\title{
B-EDCA: A New IEEE 802.11e-Based QoS Protocol for Multimedia Wireless Communications"
}

\author{
José Villalón, Pedro Cuenca, and Luis Orozco-Barbosa \\ Albacete Research Institute of Informatics, \\ Universidad de Castilla-La Mancha, \\ 02071 Albacete, Spain \\ \{josemvillalon, pcuenca, lorozco\}@info-ab.uclm.es
}

\begin{abstract}
Abtract. The IEEE 802.11e draft standard is a proposal defining the mechanisms for wireless LANs aiming to provide QoS support to time-sensitive applications. However, recent studies have shown that the IEEE 802.11e (EDCA) performs poorly when the medium is highly loaded due to the high collision rate. Even though several proposals have been proposed to address this problem, they require important changes to the current standard specifications making difficult their actual implementation. In this paper, we propose a simple QoS-aware mechanism and fully compatible with the various operation modes of the EDCA standard as well as the legacy IEEE 802.11 (DCF) scheme. Our design has been based on an in-depth analysis of the several operation modes of both standards. This should ensure full compatibility of operation: an important feature since the transition from the IEEE 802.11 to the IEEE 802.11e will take some time making more likely the existence of hybrid scenarios where both standards will have to coexist. Our simulation results show that our new scheme outperforms the EDCA and other QoS-aware schemes recently reported in the literature.
\end{abstract}

\section{Introduction}

The IEEE 802.11 WLANs [1] is being deployed widely and rapidly in many different environments including enterprise, home and public access networks. One of the most influential factors to its success is due to the development of high-speed technology enabling the deployment of multimedia applications. However, multimedia applications are not only characterized by their high bandwidth requirements, but also impose severe restrictions on delay, jitter and packet loss rate. In others words, multimedia applications require Quality of Service (QoS) support. Guaranteeing those QoS requirements in IEEE 802.11 is a very challenging task due to the QoS-unaware operation of its MAC layer. This layer uses the wireless media characterized by the difficulties faced by the signal propagation. Thus providing QoS to IEEE 802.11 has been and it is an active research area giving rise to numerous service differentiation schemes.

* This work was supported by the Ministry of Science and Technology of Spain under CICYT project TIC2003-08154-C06-02, the Council of Science and Technology of Castilla-La Macha under project PBC-03-001 and FEDER. 
Currently, the IEEE 802.11 Working Group is hardly working on the definition of the IEEE 802.11e standard [2]. The IEEE 802.11e draft is a proposal defining the mechanisms for wireless LANs aiming to provide QoS support to time-sensitive applications, such as, voice and video communications. The standardization efforts are at their final stage and it is expected that the standard will soon be publicly available.

It is expected that in the near future IEEE 802.11e-compliant interface cards will take over the WLAN market, replacing the use of legacy IEEE 802.11 interface cards in most WLAN applications. The complete migration towards the IEEE 802.11e standard will take several years given the wide scale use of legacy IEEE 802.11 in the market place today. This creates an important number of networking scenarios where legacy IEEE 802.11 based stations and IEEE 802.11e-based stations will have to interwork.

However, the ratification of the IEEE 802.11e standard is becoming a very challenging task. Many studies have shown that the IEEE 802.11e (EDCA) scheme performs poorly under heavy load conditions. The severe degradation is mainly due to high collision rates. This reason has led many researchers to design new techniques aiming to address the shortcomings of the current draft standard. However, many of the proposed techniques have overlooked two main implementation and operation issues: first, the implementation of the proposed mechanisms implies important and incompatible modifications to the IEEE 802.11e specifications in a moment in which IEEE 802.11e is at its final stage, and second, the main deficiency of these mechanisms comes from its inability to provide the QoS guarantees required by the time-constrained flows when legacy DCF based stations are present in the same scenario.

In this paper, we address the two aforementioned issues by introducing an IEEE 802.11e-compliant mechanism capable of providing QoS support even under scenarios where legacy DCF based stations are present. Our main objective has been to design a scheme able to provide the QoS guarantees required by two of the most representative time-constrained multimedia applications regardless of the channel load and under a systems configuration consisting of IEEE 802.11 and IEEE 802.11ecompliant stations. Simulation results show that our new scheme outperforms the IEEE 802.11e draft standard and some of the most relevant schemes reported in the literature. Throughout an exhaustive campaign of simulations, we have evaluated the performance of the system in terms of four metrics: throughput, access delay, delay distribution and packet loss rate.

This paper is organized as follows. Section 2 provides an overview of the IEEE 802.11 WLAN standard. In Section 3, we also describe the upcoming IEEE 802.11e QoS standard and two relevant proposals recently reported in the literature aiming to improve the performance of the IEEE 802.11e standard. In Section 4, we present our new IEEE 802.11e based QoS mechanism. In Section 5, we carry out a comparative performance evaluation when supporting different services, such as, voice, video, best-effort, background and in the presence of traffic generated by legacy DCF based stations. Finally, Section 6 concludes the paper.

\section{Overview of IEEE 802.11 WLAN}

The IEEE 802.11 MAC sub-layer [1] defines two medium access coordination functions, the Distributed Coordination Function (DCF) and the optional Point 
Coordination Function (PCF). DCF is the basic access function for IEEE 802.11 and is based in a Carrier Sense Multiple Access with Collision Avoidance (CSMA/CA) algorithm together with a contention (backoff) algorithm. PCF uses a centralized polling method requiring a node to play the role of Point Coordinator (PC). The PC cyclically polls the stations to give them the opportunity to transmit. In the following, we restraint our description to the DCF mechanism whose mode of operation may affect the ability of the upcoming IEEE 802.11e (EDCA) standard to provide QoS guarantees.

A station operating under the DCF scheme should first sense the state of the channel before initiating a transmission. A station may start to transmit after having determined that the channel is idle during an interval of time longer than the Distributed InterFrame Space (DIFS). Otherwise, if the channel is sensed busy, once the transmission in course finishes and in order to avoid a potential collision with other active (waiting) stations, the station will wait a random interval of time (the Backoff_Time) before starting to transmit. As long as no activity is detected in the channel, a backoff counter, initially set to Backoff_Time, is decremented on an aSlotTime by aSlotTime basis. Whenever activity is detected, the backoff counter is frozen and reactivated once again when the channel has remained idle during an interval of time longer than DIFS. The station will be able to begin transmission as soon as the backoff counter reaches zero. In case of an unsuccessful transmission, the station will have a finite number of attempts, using a longer backoff time after each attempt.

Even though DCF is a simple and effective mechanism, DCF can neither support QoS nor guarantee to meet the multimedia applications requirements. It is for this reason that many researchers have proposed techniques the provisioning of QoS mechanisms into the DCF mode of operation. The description of such mechanisms is out of the scope of this work. An overview of many of the different QoS enhancements mechanisms for the IEEE 802.11 standards can be found in [3]. In that work, the authors have summarized and classified a large number of the proposed techniques. A comparative performance evaluation of some of them can also be found in [4], [5], [6].

\section{The IEEE 802.11e Draft Standard}

The IEEE 802.11e draft standard [2] aims to specify the mechanisms enabling the provisioning of QoS guarantees in IEEE 802.11 WLANs. In the IEEE 802.11e standard, distinction is made among those stations not requiring QoS support, known as $n Q S T A$, and those requiring it, QSTA. In order to support both Intserv and DiffServ QoS approaches in an IEEE 802.11 WLAN, a third coordination function is being added: the Hybrid Coordination Function (HCF). The use of this new coordination function is mandatory for the QSTAs. HCF incorporates two new access mechanisms: the contention-based Enhanced Distributed Channel Access (EDCA), known in the previous drafts as the Enhanced DCF (EDCF) and the HCF Controlled Channel Access (HCCA). In the HCCA mechanism a central node is used for coordinating the access to the channel: the Hybrid Coordinator (HC). When the HC takes control over the channel during the Contention Period $(\mathrm{CP})$, it is said that a 
Controlled Access Phase (CAP) has been generated. It is worth noting that the HC should at all times hold the highest priority allowing it to initiate the CAP.

One main feature of HCF is the definition of four Access Categories (AC) queues and eight Traffic Stream (TS) queues at MAC layer. When a frame arrives at the MAC layer, it is tagged with a Traffic Priority Identifier (TID) according to its QoS requirements, which can take values from 0 to 15 . The frames with TID values from 0 to 7 are mapped into four AC queues using the EDCA access rules. The frames with TID values from 8 to 15 are mapped into the eight TS queues using the HCF controlled channel access rules. The TS queues provide a strict parameterized QoS control while the AC queues enable the provisioning of multiple priorities. Another main feature of the HCF is the concept of Transmission Opportunity (TXOP), which defines the transmission holding time for each station.

EDCA has been designed to be used with the contention-based prioritized QoS support mechanisms. In EDCA, two main methods are introduced to support service differentiation. The first one is to use different IFS values for different ACs. The second method consists in allocating different $\mathrm{CW}$ sizes to the different ACs. Each $\mathrm{AC}$ forms an EDCA independent entity with its own queue and its own access mechanism based on an DCF-like mechanism with its own Arbitration Inter-Frame Space defined by $A I F S[A C]=S I F S+A I F S[A C] \times$ SlotTime and its own CW[AC] $(C W \min [A C] \leq C W[A C] \leq C W \max [A C])$, where AIFSN[AC] is the Arbitration Inter Frame Space Number. If an internal collision arises among the queues within the same QSTA, the one having higher priority obtains the right to transmit. It is said that the queue getting the right to access to the channel obtains a transmission opportunity (TXOP). The winning queue can then transmit during a time interval whose length is given by TXOPLimit.

\subsection{QoS Enhancements to the IEEE 802.11e}

Many on-going research efforts are focusing on the evaluation of the IEEE 802.11e draft standard. Many studies have revealed that the poor performance exhibited by the draft standard is mainly due to the high collision rates encountered when a large number of stations attempt to access the channel. Numerous proposals have been reported in the literature aiming to overcome this main drawback. In the following, we undertake the analysis of two of the most prominent ones.

The Fast Collision Resolution Mechanism FCR [7] aims to shorten the backoff period by increasing the contention window sizes of all active stations during the contention resolution period. To reduce the number of wasted (idle) slots, the FCR algorithm assigns the shortest window size and idle backoff timer to the station having successfully transmitted a packet. Moreover, when a station detects a number of idle slots (static backoff threshold), it starts reducing the backoff timer exponentially, instead of linearly as specified by the EDCA draft standard. To address the provisioning of QoS mechanisms, the authors further introduce an enhanced version of the FCR algorithm, namely, the Real Time Fast Collision Resolution (RTFCR) [7] algorithm. In this algorithm, the priorities are implemented by assigning different backoff ranges based on the type of traffic. In their study, the authors have considered three main traffic types: voice, video, and best-effort (data) traffic. 
Under this scheme, voice packets hold the highest priority to access the channel by setting $\mathrm{CW}=\mathrm{CW}_{\min }$. All the other flows have to wait, at least, eight backoff slots before being allowed to gain access to the channel. The video traffic is assigned the second highest priority by using a smaller maximum contention window size than the one assigned to the best-effort data traffic.

The Adaptive EDCF Mechanism (AEDCF) [8] is another relevant mechanism recently reported in the literature. In [8], the authors state that the probability of collision increases is due to the re-setting of $C W[A C]$ to $C W m i n[A C]$ after a successful transmission in the presence of multiple stations contending for the channel. Taking this fact into account, they have proposed decreasing the $C W[A C]$ by multiplying by a factor lower than 0.8 after a successful transmission; the actual value of the factor will depend on the collision rate suffered by the AC. In [9], the same authors go a step further by introducing a new scheme called Adaptive Fair EDCF (AFEDCF) that improves AEDCF and FCR mechanisms. This mechanism uses an adaptive fast collision resolution mechanism (similar to the FCR mechanism) when the channel is sensed idle. In contrast with the FCR mechanism, AFEDCF computes an adaptive backoff threshold for each priority level by taking into account the channel load.

However, the main deficiency of these mechanisms comes from its inability to provide the proper QoS to the video service in scenarios comprising legacy DCFbased and IEEE 802.11e stations. This is due to the fact that, under theses schemes, the video packets have always to wait for a minimum of eight backoff slots in order to comply with the highest priority assigned to the voice traffic. Under these schemes, the presence of voice and DCF stations may even result in starvation to the video flows. Moreover, the implementation of these mechanisms implies that the stations have to monitor the channel conditions in order to dynamically tune up the actual values of the key system parameters, such as the threshold and window size.

Taking into account these observations, in the next section, we propose a new IEEE 802.11e based QoS mechanism capable of providing QoS support to the video service even in the presence of legacy IEEE 802.11 (DCF) based stations.

\section{B-EDCA: A New IEEE 802.11e Based QoS Mechanism}

Due to the fact that the IEEE 802.11e interface cards will take over the WLAN market, replacing the use of legacy IEEE 802.11 interface cards in most WLAN applications, an important number of networking scenarios will consist of a hybrid configuration comprising legacy IEEE 802.11-based stations and IEEE 802.11e-based stations. Under these scenarios, EDCA, RT-FCR and AFEDCF perform poorly, especially they are unable to provide the QoS required by the video traffic.

Based on limitations of these mechanisms, we propose a new IEEE-802.11e based QoS mechanism compatible with the IEEE 802.11e specifications and capable of providing QoS support, particularly to video applications.

Bearing in mind that the DCF and EDCA mechanisms may have to interwork, the standard committee has set up the system parameters given in Table I. These values have been set up in order to ensure compatibility between both services and that the EDCA mechanism has to be able to provide QoS guarantees to time-constrained 
applications, namely voice and video traffic. As shown in Table I, the EDCA mechanism makes the use of a smaller contention window for the voice and video applications.

Based on the results obtained in one of our previous studies [10], we have found out that the IFS (denoted AIFS in the EDCA draft standard) is the most important and critical parameter enabling the provisioning of QoS to multimedia applications. This is particular true when a large number of stations attempt to gain access to the channel, since under these conditions, the stations will often have to stop decrementing their backoff counters. Recall that every time that a station stops decrementing its counter, the station must wait an AIFS before resuming the count down.

Table 1. Parameter settings specified in standards [1], [2]

\begin{tabular}{|c|c|c|c|c|}
\hline & $\mathbf{A C}$ & IFS & $\mathbf{C W}_{\min }$ & $\mathbf{C W}_{\max }$ \\
\hline DCF & - & $2 \times$ Slot_time + SIFS & 31 & 1023 \\
\hline \multirow{4}{*}{ 导 } & Vo & $2 \times$ Slot_time + SIFS & 7 & 15 \\
\hline & $\mathrm{Vi}$ & $2 \times$ Slot_time + SIFS & 15 & 31 \\
\hline & $\mathrm{Be}$ & $3 \times$ Slot_time + SIFS & 31 & 1023 \\
\hline & $\mathrm{Bk}$ & $7 \times$ Slot_time + SIFS & 31 & 1023 \\
\hline
\end{tabular}

One possible solution will be to set up $A I F S=1$ for the voice and video applications. In this way, they will increase their chances to gain access to the channel. However, setting up AIFS $=1$ to these two services is incompatible with the HCCA. As already explained, the HC should be able to take the control of the channel at any time. This is to say, the HCCA should hold the highest priority over all the services to be supported by the standard.

In order to introduce our proposal, we take a closer look at the mode of operation of the DCF and EDCA schemes, and particularly on the role played by the IFS (AIFS) parameter. The IFS (AIFS) is used in the following two cases:

1. In the Idle state. when the station becomes active has to sense the channel during an interval whose length is determined by IFS: If the channel is sensed free, the station can initiate the packet transmission. Otherwise, the station executes the backoff algorithm.

2. In every transfer from the Defer state to the Backoff state. In other words, every time after having sensed the channel free during an interval of length IFS.

According to the current DCF and EDCA standards, the same values for the IFS parameter should be used regardless of the state in which the station is (see Table 1). Based on the previous observation, we then propose to use a different set of IFS values depending on the state in which the station is. We have however to ensure not to compromise the operation of the $\mathrm{HCF}$, and in particular to ensure that it holds at all times the highest priority. We then propose the following parameter setting:

1. In the Idle state. The stations will use the IFS values as specified in the IEEE 802.11e draft standard (see Table I) including the Hybrid Coordination Function. This also ensures compatibility with the IEEE 802.11 (DCF) mechanism. 
2. In every transfer from the Defer state to the Backoff state, we propose to use a different parameter, equivalent to the IFS, denoted from now on by BIFS. We then propose setting up this parameter to one, i.e., BIFS $=1$, for the voice and video services. In this way, we improve considerably the performance of voice and video applications, increasing their priorities with respect to other flows (included the traffic generated by DCF-based stations). This setting also ensures that the HC will keep the highest priority. According to this mechanism, the stations must wait at least one additional slot during the backoff procedure before being allowed to transmit since the backoff interval is set within the $[1, \mathrm{CW}+1]$ range. In turn, the $\mathrm{HC}$ is allowed to take the control at the end of the IFS. To improve further the provisioning of QoS guarantees to the time-constrained applications when the network is highly loaded, we propose increasing the assigned value to BIFS used by the Best-Effort traffic, with respect to the specified in [2]. We then propose using the set of values for BIFS to 1-1-4-7 for voice, video, best-effort and background traffics, respectively.

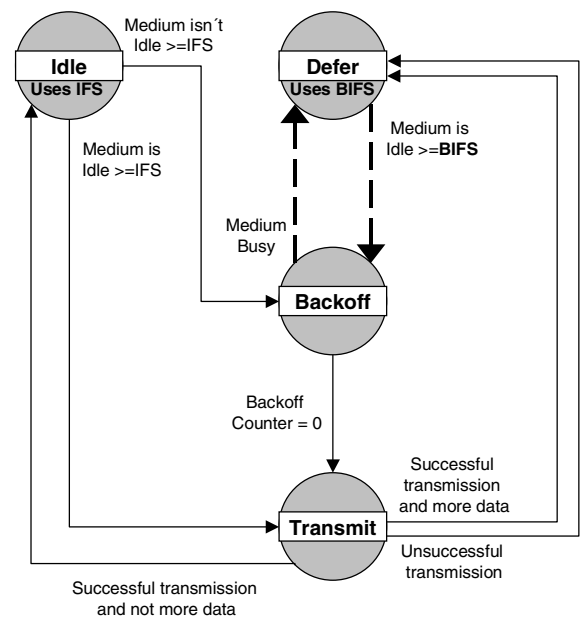

Fig. 1. B-EDCA Proposed Mechanism

In Figure 1, we have explicitly indicated the instances where the BIFS parameter should be used. This is essentially the major change with respect to the current EDCA standard. Our proposal essentially reduces to the minimum acceptable value, the waiting time required to continue decrementing the backoff counter used by the timeconstrained applications. This minimum value is fully compatible with the operation modes of the DCF and HCCA functions.

\section{Performance Evaluation}

In this section, we carry out a performance analysis of our proposed mechanism. We show that the performance of EDCA can be considerably improved by using the 
compatible B-EDCA mechanism. In this part of our study, we compare the performance of our proposed scheme with the EDCA, RT-FCR, AFEDCF mechanisms by considering a scenario of a wireless LANs comprising IEEE 802.11based stations and stations supporting one of the QoS-aware mechanisms under study. Throughout our study, we have made use of the OPNET Modeler tool 10.0 [11].

\subsection{Scenario}

In our simulations, we model an IEEE 802.11b wireless LAN cell comprising legacy DCF-based stations and stations implementing one of the four QoS-aware mechanism stations under consideration. The QoS-aware mechanism based stations support four different types of services: voice (Vo), video (Vi), best-effort (BE) and background (BK). This classification is in line with the IEEE802.1D standard specifications. The DCF based stations support data traffic. We assume the use of a wireless LAN consisting of several wireless stations and an access point connected to a wired node that serves as sink for the flows from the wireless domain. All the stations are located within a Basic Service Set (BSS), i.e., every station is able to detect the transmission from any other station.

Each wireless station operates at $11 \mathrm{Mbit} / \mathrm{s}$ IEEE $802.11 \mathrm{~b}$ mode and transmits a single traffic type to the access point. We assume the use of constant bit-rate voice sources encoded at a rate of $16 \mathrm{kbits} / \mathrm{s}$ according to the G.728 standard [12]. The voice packet size has been set to 168 bytes including the RTP/UDP/IP headers. For the video applications, we have made use of the traces generated from a variable bitrate H.264 video encoder [13]. We have used the sequence mobile calendar encoded on CIF format at a video frame rate of 25 frames/sec. The average video transmission rate is around $480 \mathrm{kbits} / \mathrm{s}$ with a packet size equal to 1064 bytes (including RTP/UDP/IP headers). The best-effort, background and DCF traffics have been created using a Pareto distribution traffic model. The average sending rate of besteffort and background traffic is $128 \mathrm{kbit} / \mathrm{s}$, using a 552 bytes packet size (including TCP/IP headers). The average sending rate of DCF traffic is $256 \mathrm{kbit} / \mathrm{s}$, using a 552 bytes packet size (including TCP/IP headers). All traffic sources are randomly activated within of the interval $[1,1.5]$ seconds from the start of the simulation. We have simulated two minutes of operation for each given scenario.

For all the scenarios, we have assumed that one fifth of the stations support one of the five kinds of services: voice, video, BE, BK and DCF applications. We start by simulating a WLAN consisting of five wireless stations (each one supporting a different type of traffic). We then gradually increase the Total Offered Load of the wireless LAN by increasing the number of stations by five. In this way, the stations are always incorporated into the system in a ratio of $1: 1: 1: 1: 1$ for voice, video, $\mathrm{BE}$, BK and DCF, respectively. We increase the number of stations 5 by 5 starting from 5 and up to 40. In this way, the normalized offered load is increased from 0.14 up to 1.12. By exceeding the channel capacity, we should be able to evaluate the effectiveness of the QoS-aware mechanisms on guaranteeing the QoS required by the time-constrained applications. When choosing the parameter settings to use for the DCF and EDCA mechanisms under study, we have used the settings recommended by the standards [1], [2] (see Table I). The parameter settings used for the RT-FCR and AFEDCF mechanisms under study have been taken from references [7] and [9], 
respectively. The parameter settings for the B-EDCA mechanism have been defined by following the guidelines provided in Section 4.

For the purpose of our performance study, the four metrics of interest are: throughput, media access delay, delay distribution and packet loss rate. To be able to compare the results at different loads (traffic patterns of different applications), we have preferred plotting the normalized throughput rather than the absolute throughput. The normalized throughput is calculated as the percentage of the offered load actually delivered to destination. In order to limit the delay experienced by the video and voice applications, the maximum time that video packet and voice packet may remain in the transmission buffer has been set to $100 \mathrm{~ms}$ and $10 \mathrm{~ms}$, respectively. These time limits are in line with the values specified by the standards and in the literature. Whenever a video or voice packet exceeds these upper bounds, it is dropped. The loss rate due to this mechanism is given by the packet loss rate due to deadline. Our measurements started after a warm-up period allowing us to collect the statistics under steady-state conditions. Each point in our plots is an average over thirty simulation runs, and the error bars indicate the $95 \%$ confidence interval.

\subsection{Results}

Figure 2 shows the normalized throughput obtained for the Vo, Vi, BE and BK services when making use of each one of the four mechanisms being considered. Figure 2a shows that B-EDCA mechanism outperforms the EDCA mechanism in providing a better service to the voice traffic. This shows that by reducing the effectiveness of setting the BIFS parameter to one. The figure also shows that the RTFCR and AFEDCF mechanisms obtain the best results for the voice traffic. This is due to the fact that, under theses schemes, the highest priority is given to the voice traffic, all the other traffic types have to wait a minimum of eight backoff slots. However, under these schemes, the presence of voice and DCF stations produces starvation in the video flows, see Figure $2 \mathrm{~b}$. For video traffic, under RT-FCR and AFEDCF mechanisms, when the load exceeds 0.5, the throughput of the video traffic quickly decreases. The decrease on the video throughput is mainly due to the fact that under the RT-FCR and AFEDCF mechanisms, the DCF based stations have a higher priority than the one given to the video stations, see Figure 2e. Figure $2 b$ also shows that B-EDCA obtain the best results for the video traffic. Again, for the case of the video traffic, the B-EDCA mechanism outperforms the EDCA mechanism. In the case of the $\mathrm{BE}$ and $\mathrm{BK}$ traffics (figures $2 \mathrm{c}$ and $2 \mathrm{~d}$ ), these are severely affected as the network load is increased. Figure $2 \mathrm{f}$ shows the overall throughput for all the services under study. It is clear that the B-EDCA exhibits the highest normalized throughput. This is due to the reduction of the collision rate with respect to EDCA mechanism, and to the fact that in the RT-FCR and AFEDCF mechanisms, all the flows (except voice) must wait eight additional backoff slots.

These phenomena also explain the access delay performance. Figure 3 shows the mean access delay per voice and video service classes. Figure 3 a shows that the BEDCA reduces up to $50 \%$ the mean access delay experienced by the voice traffic when using the EDCA mechanism. Figure $4 \mathrm{~b}$ shows that the B-EDCA scheme exhibits the best results for the video service. It can also be observed that the mean 
access delays for RT-FCR and AFEDCF mechanisms are very close to the video deadlines; this in turn translates in a high packet loss rate (Figure 5).

Figure 4 shows the cumulative distribution function of the access delay for all mechanism operating at a load close to 0.80. Figure $4 \mathrm{a}$ shows that B-EDCA mechanism outperforms the EDCA mechanism for the voice traffic. Figure $4 \mathrm{~b}$ also shows that B-EDCA obtain the best results for the video traffic.

Figures $5 \mathrm{a}$ and $5 \mathrm{~b}$ depict the packet loss rate due to the missing of the transmission deadline for the voice and video traffic services, respectively. The B-EDCA scheme provides the best results for the video traffic. The B-EDCA scheme is able to ensure the proper transmission of the video traffic even at loads as high as 0.8 .

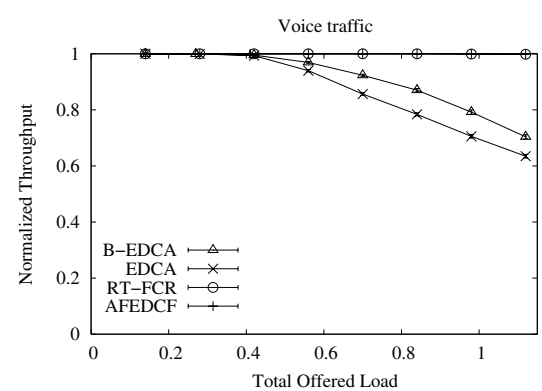

(a)

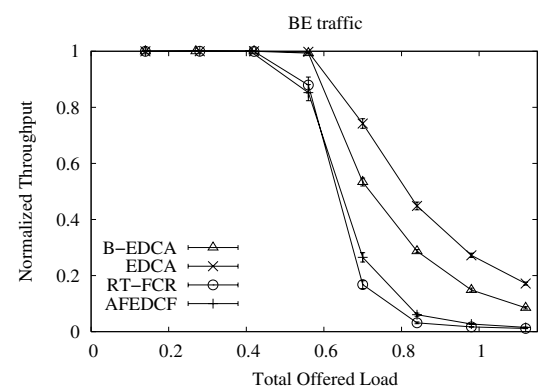

(c)

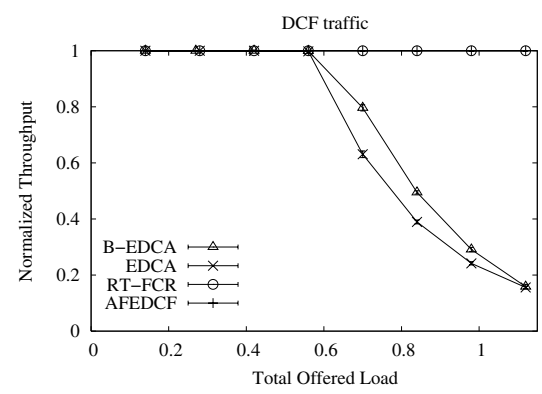

(e)

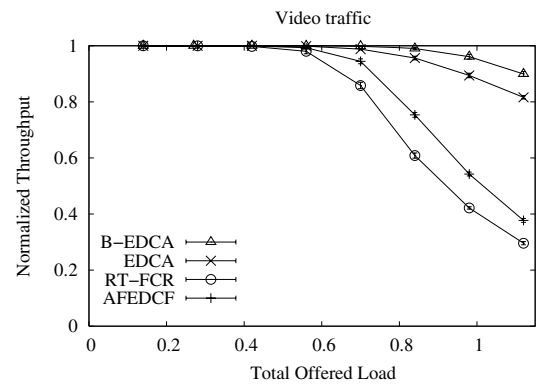

(b)

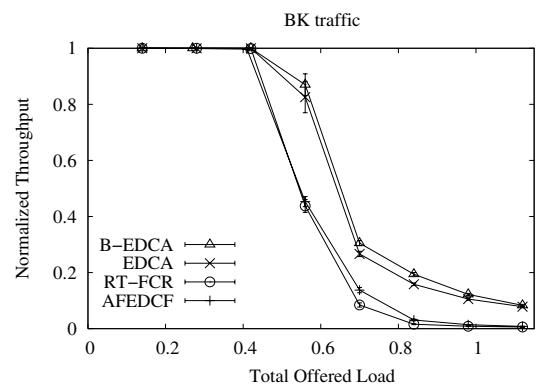

(d)

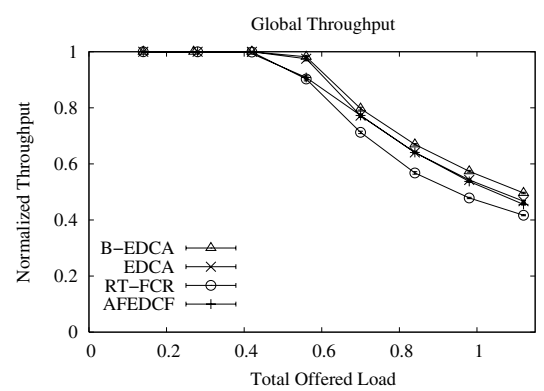

(f)

Fig. 2. Average Normalized Throughput: a) Voice, b) Video, c) Best-Effort d) Background e) DCF Traffic and f) Total Traffic 


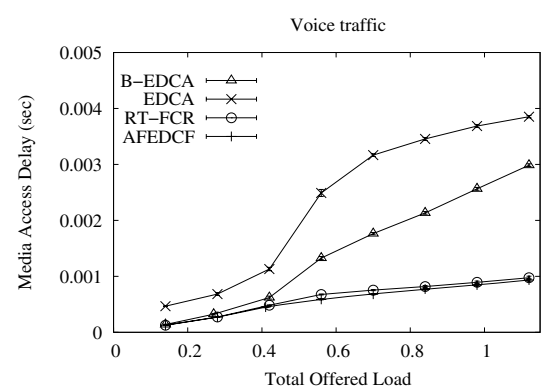

(a)

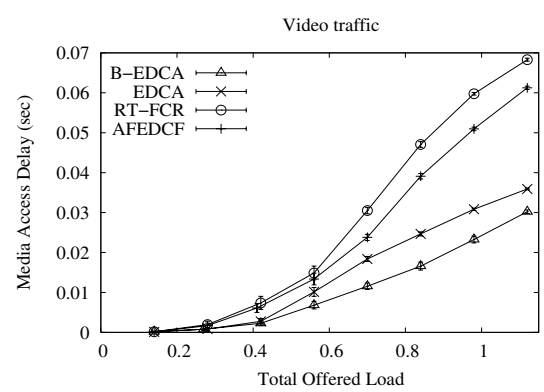

(b)

Fig. 3. Average Access Delay: a) voice, b) video

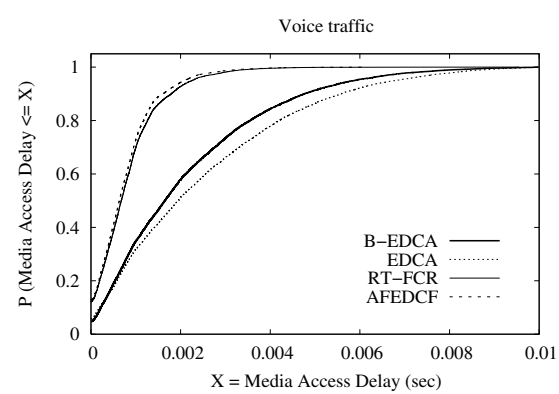

(a)

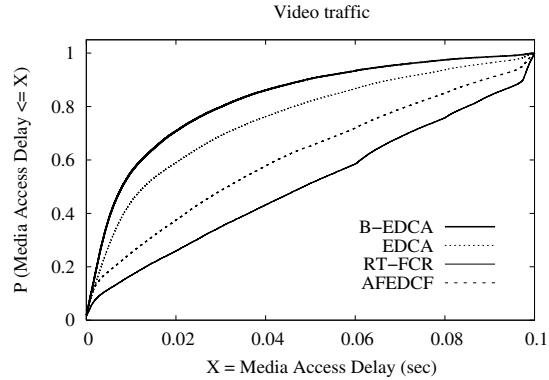

(b)

Fig. 4. Cumulative Distribution (CDF) of the Access Delays: a) voice, b) video

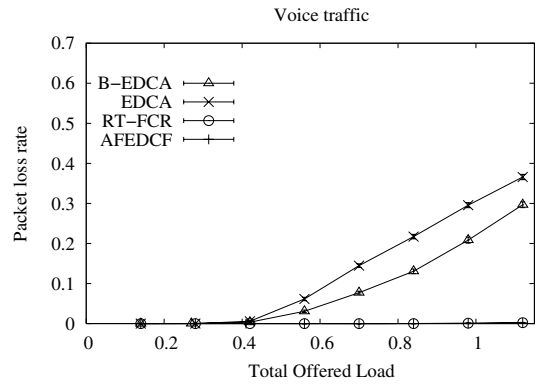

(a)

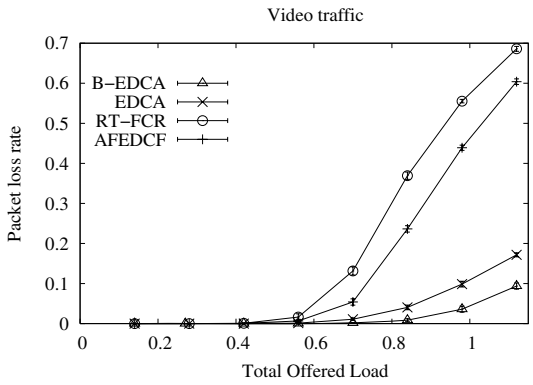

(b)

Fig. 5. Packet Loss Rate due to Deadline: a) voice and b) video

\section{Conclusions}

In this paper, we have proposed a new IEEE 802.11e based QoS protocol design capable of providing QoS support in environments where legacy DCF based stations may also be present. Our proposal has been based in using the minimum waiting time 
necessary to continue decrementing the backoff counter of the multimedia flows. Furthermore, our proposal complies with the HCF operation proposed by the IEEE 802.11e standards. Our results obtained have shown that B-EDCA mechanism outperforms the EDCA mechanism and two other relevant mechanisms reported in the literature.

\section{References}

1. LAN MAN Standards Committee of the IEEE Computer Society, ANSI/IEEE Std 802.11, "Part 11: Wireless LAN Medium Access Control (MAC) and Physical Layer (PHY) Specifications", 1999 Edition.

2. IEEE 802 Committee of the IEEE Computer Society, IEEE P802.11e/D13.0 Draft Amendment to IEEE Std 802.11, "Part 11: Wireless LAN Medium Access Control (MAC) and Physical Layer (PHY) Specifications: Medium Access Control (MAC) Quality of Service (QoS) Enhancements", April 2005.

3. F. Mico, P. Cuenca and L.Orozco Barbosa "QoS Mechanisms for IEEE 802.11 Wireless LANs”. Lecture Notes in Computer Science. Vol. 3079. pp. 609-623, 2004.

4. J. Villalón, P. Cuenca, L. Orozco-Barbosa. "QoS Provisioning Mechanisms for IEEE 802.11 WLAN: A Performance Evaluation". Proceedings of 10th IFIP International Conference on Personal Wireless Communications. Colmar, August 2005.

5. A. Lindgren, A. Almquist and O. Schelén, "Quality of Service Schemes for IEEE 802.11 Wireless LANs - An Evaluation", Journal of Special Topics in Mobile Networking and Applications (MONET), Vol. 8, No. 3, pp. 223-235, June 2003.

6. W. Pattara-Atikom, P. Krishnamurthy and S. Banerjee, "Distributed Mechanisms for Quality of Service in Wireless LANs", IEEE Wireless Communications, Vol. 10, No. 3, pp. 26-34, June 2003.

7. Y. Kwon, Y. Fang and H. Latchman, "Design of MAC Protocols with Fast Collision Resolution for Wireless Local Area Networks". IEEE Transactions on Wireless Communications, Vol. 3, No.3. pp. 793-807, May 2004.

8. L. Romdhani, Q. Ni and T. Turletti, "Adaptive EDCF: Enhanced Service Differentiation for IEEE 802.11 Wireless Ad-Hoc Networks", in Proceedings of IEEE WCNC, New Orleans, pp. 1373-1378. March 2003.

9. M. Malli, Q. Ni, T. Turletti and C. Barakat "Adaptive Fair Channel Allocation for QoS Enhancement in IEEE 802.11 Wireless LANs", Proceedings of IEEE ICC, Paris, June 2004.

10. J. Villalón, P. Cuenca, L. Orozco-Barbosa, "On the Effectiveness of IEEE 802.11e QoS Support in Wireless LAN: A Performance Analysis". Lecture Notes in Computer Science. Vol. 3726. pp. 605-616, 2005.

11. Opnet.Technologies.Inc. OPNET Modeler 10.0, 1987-2004. http://www.opnet.com

12. ITU-T Recommendation G.728, "Coding of Speech at $16 \mathrm{kbit} / \mathrm{s}$ using Low-Delay Code Excited Linear Prediction", Std., September 1992.

13. ITU-T Recommendation H.264, "Advanced Video Coding For Generic Audiovisual Services". May 2003. 